\title{
Coherent cavity ring down spectroscopy
}

\author{
Gerard Meijer, Maarten G.H. Boogaarts, Rienk T. Jongma, David H. Parker \\ Department of Molecular and Laser Physics, University of Nijmegen, Toernooiveld, 6525 ED Nijmegen, The Netherlands
}

and

\author{
Alec M. Wodtke ${ }^{1}$ \\ Department of Chemistry, University of California, Santa Barbara, CA 93106, USA
}

Received 29 October 1993

\begin{abstract}
In a cavity ring down experiment the multi-mode structure of a short resonant cavity has been explicitly manipulated to allow a high spectral resolution, which is advantageous for the overall detection sensitivity as well. Coherent cavity ring down spectroscopy is performed around $298 \mathrm{~nm}$ on $\mathrm{OH}$ in a flame.
\end{abstract}

Direct detection of molecular absorption of light from a laser beam is preferable to other spectroscopic methods, since it can still be applied with success even when the molecule's excited state does not fluoresce (a prepequisite of LIF) or cannot be ionized (a prerequisite of REMPI). Application of direct absorption techniques in high pressure samples, e.g. flames and plasmas, is also advantageous since Beer's law can be used to extract quantitative $a b$ solute concentration data which is nearly impossible by LIF due to the difficulties associated with collisional quenching of the fluorescing state. Furthermore, unlike other techniques, for example degenerate four wave mixing, direct absorption is a linear spectroscopic technique both in molecular number density and absorption strength. It is therefore more easily quantifiable and will scale more favorably over large variations of molecular absorption strength and concentration.

In order to make sensitive $\Delta I / I$ absorption measurements, one must be able to distinguish laser beam intensity fluctuations from molecular absorptions. This problem is particularly daunting for pulsed las-

\footnotetext{
1 National Science Foundation Presidential Young Investigator, Alfred P. Sloan Research Fellow, Camille and Henry Dreyfus Teaching-Scholar.
}

ers whose shot-to-shot fluctuations are typically $5 \%$. Although these fluctuations can be normalized out to some extent, absorptions on the order of $10^{-3}$ are needed to yield an observable signal [1]. Cavity ring down spectroscopy (CRDS) was introduced as a sensitive gas phase direct absorption technique by O'Keefe and Deacon [2] in 1988. The method is based upon the measurement of the rate of absorption rather than the magnitude of absorption of a light pulse confined within a closed optical cavity. By measuring the cavity decay time, i.e. the exponential time constant which describes the time-dependent probability for loss of a photon from the cavity modes of a stable resonator, the aforementioned problems associated with pulsed lasers are circumvented. This can be easily appreciated if one realizes that the cavity decay time $\tau$ is independent of the amplitude of the light circulating inside the cavity

$$
\tau=\frac{d}{c(1-R+\sigma n l)},
$$

where $d$ is the optical length of the cavity, $c$ the speed of light, $R$ the averaged reflectivity of the two mirrors and $\sigma$ is the absorption cross section of the molecular sample with a density $n$ (per quantum state) and length $l$.

O'Keefe and Deacon showed that absorption losses 
$\Delta I / I$ of about 1 ppm per pass can be detected by CRDS [2]. Since then O'Keefe et al. demonstrated that this technique can be used to perform sensitive absorption spectroscopy in a molecular jet expansion [3]. Romanini and Lehmann have used CRDS for a spectroscopic study of the stretching overtones in HCN and established that it is of comparable sensitivity to photoacoustic spectroscopy [4]. Yu and Lin have recently shown that CRDS can be used for quantitative kinetics measurements as well [5].

Up until now it has been believed that care should be taken to use a light source with a coherence length short enough to prevent interference and the concommitant buildup of cavity mode structure in the ring down cavity [2]. If cavity mode structure is allowed to build up, certain problems can arise. For example, narrow molecular absorption features might fall between the cavity modes and these features would then be missing from the spectrum. Even worse, since the ring down cavity is normally unstabilized, the spectra might be irreproducible. In addition, a narrowband laser might not couple into the cavity if it happened to sit between two transmission features of the cavity. Considering all of this, a typical setup uses a 1-2 m long cavity and a short coherence length, i.e. a broad band, probe laser. In all cavity ring down experiments reported to date, the spectral resolution of the short coherence length probe laser has been limited to about $1 \mathrm{~cm}^{-1}$. Pressure broadening can then be used to match the width of the molecular absorption lines to the laser bandwidth [4].

In many cases, however, molecular absorption features are much narrower than $1 \mathrm{~cm}^{-1}$. When this is the case, an apparatus as described above will suffer a sensitivity loss in addition to an information loss. It is unclear, in light of the above discussion, how this promising technique should be extended to higher resolution. Another limitation of CRDS has been its reliance on very high reflectivity mirrors, typically $R=0.9999$ or better. This essentially eliminates the UV part of the spectrum for CRDS since at wavelengths less than about $450 \mathrm{~nm}$, mirrors with $R \geqslant 0.997$ are not available. This is a particularly gauling problem since it is in the UV that pulsed lasers are most useful.

Another important question is whether or not CRDS can be used on less well-defined spectral ob- jects, such as flames or plasmas, where its quantitative properties could be exploited. It is not clear from the work presently in the literature if thermal lensing, particle scattering and turbulent fluctuations will destroy the utility of CRDS.

In this Letter, we report the use of coherent cavity ring down spectroscopy (COCRDS), a form of CRDS that uses a long coherence length laser and a short cavity in order to obtain high spectral resolution. The improved spectral resolution also means a gain in absolute detection sensitivity. In addition, this Letter reports the first extension of CRDS to the UV. Even in this region of the spectrum, where mirrors with a reflectivity of $R \approx 0.995$ had to be used, $10 \mathrm{ppm}$ (per pass) absorption sensitivity is shown to be possible. In particular we have obtained absorption spectra of rotationally (up to $N^{\prime \prime}=27$ ) and vibrationally ( $v^{\prime \prime}=1$ and 2 ) excited $\mathrm{OH}$ in a $\mathrm{CH}_{4} /$ air flame near $298 \mathrm{~nm}$ via COCRDS. A test of the MaxwellBoltzmann law was thereby performed and the quantitative nature of COCRDS was demonstrated.

The key to these experiments is the design of the cavity. We have used 1 inch diameter plano-concave mirrors with $10 \mathrm{~cm}$ radii of curvature, coated for $\geqslant 99.5 \%$ normal incidence reflectivity at $308 \mathrm{~nm}$, i.e. standard $\mathrm{XeCl}$ excimer laser high reflectors (Laseroptik $\mathrm{GmbH}$, Garbsen, Germany). The substrate material was Herasil. For measurements on $I_{2}$ described below we used similar mirrors coated for $\geqslant 99.5 \%$ reflectivity between 540 and $640 \mathrm{~nm}$. The mirrors were mounted inside a vacuum chamber and could be adjusted with micrometer screws from the outside.

The central feature of COCRDS is that it intentionally manipulates the cavity mode structure, which has been avoided in other work, in order to allow higher resolution. To demonstrate this we have measured the cavity mode structure of the aforementioned "308 nm cavity" using an intracavity frequency-doubled ring-dye laser delivering $1 \mathrm{~mW}$ of $318 \mathrm{~nm}$ radiation with a $5 \mathrm{MHz}$ bandwidth. The slightly diverging $2 \mathrm{~mm}$ diameter beam is coupled into the cavity along the axis and the cavity transmission is measured while the laser is continuously scanned over $3 \mathrm{GHz}$. The transmission of the cavity is shown in fig. 1 as a function of the mirror separation $d$. At the confocal setting, $d=10 \mathrm{~cm}$, the expected simple transmission spectrum is obtained (fig. 


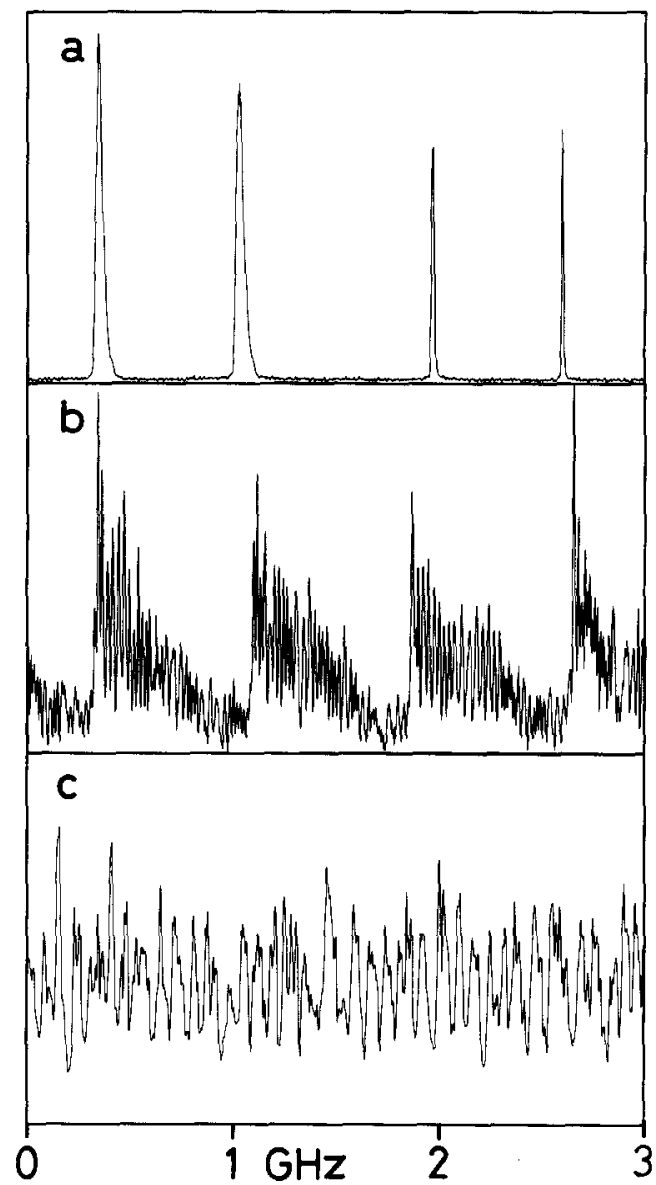

Fig. 1. Mode-spectrum of the ring down cavity for three different mirror separations $d$ : (a) $d=10.0 \mathrm{~cm}$; (b) $d=10.2 \mathrm{~cm}$; (c) $d=11.5 \mathrm{~cm}$. A rapid congestion of the spectrum is seen when the cavity is detuned from confocal. The spectra are measured by monitoring the transmission of the cavity while a narrowband ( $\leqslant 5 \mathrm{MHz}$ ) cw UV laser is scanned over $3 \mathrm{GHz}(6 \mathrm{GHz} / \mathrm{min}$ scan-rate) around $318 \mathrm{~nm}$.

1a). The distance between adjacent longitudinal modes is $1.5 \mathrm{GHz}$, whereas the transverse modes are only $750 \mathrm{MHz}$ apart, every other one being degenerate with a longitudinal mode. The combination of these modes gives the expected $c / 4 d$ mode spacing. The cavity modes in fig. 1 a are not exactly equidistant due to the drift of the unstabilized ring down cavity during the recording time of the spectrum. The minimum width of individual modes is found to be around $10 \mathrm{MHz}$, a combination of the bandwidth of the $\mathrm{cw}$ laser and the finesse of the ring down cavity at the probe wavelength. If one would try COCRDS with this cavity the "missing lines" and "input coupling" problems mentioned above are obvious.

In fig. $1 \mathrm{~b}$ the mode-structure of the cavity is shown when the mirror separation is slightly removed from the confocal separation, $d=10.2 \mathrm{~cm}$. The transverse mode spacing is no longer exactly half the longitudinal mode spacing and because there are clearly a large number of transverse modes excited by the laser, a more complex mode spectrum is obtained. An approximately $750 \mathrm{MHz}$ periodicity can still be recognized in the spectrum. For a mirror separation of $11.5 \mathrm{~cm}$, as was used in the COCRDS experiments described later, the very dense mode spectrum shown in fig. $1 \mathrm{c}$ is obtained. It should be explicitly noted that there is no part of the spectrum where there is no transmission of the narrowband laser.

It is important to emphasize that all of these mirror spacings correspond to stable resonators as given by the condition $0<d<R_{1}$ or $R_{2}<d<\left(R_{1}+R_{2}\right)$ for a stable resonator consisting of two mirrors with radii $R_{1}$ and $R_{2} \geqslant R_{1}$ [6]. The important point is that one can use a short cavity which subtends a large solid angle to establish a superposition of transverse and longitudinal cavity modes which is nearly a continuum if a mirror separation away from the confocal $d=\frac{1}{2}\left(R_{1}+R_{2}\right)$ setting is used. In a cavity such as that associated with fig. 1c a large number of transverse modes are readily set up and, with the distance chosen as described, these transverse modes fall spectrally in between the longitudinal cavity modes, thereby filling up the mode spectrum of the cavity. By establishing a "quasi-continuum" of cavity modes one guarantees that there will always be several cavity modes underneath a spectral transition and that even a narrowband laser will be efficiently coupled into the cavity.

For the COCRDS experiments about $10 \mu \mathrm{J}$ of the output of a pulsed dye laser (Spectra Physics, PDL3) pumped by the duubled output of a Nd:YAG (Spectra Physics, GCR-11) laser was used. This afforded a spectral resolution of $0.05 \mathrm{~cm}^{-1}$ in the visible and $0.1 \mathrm{~cm}^{-1}$ when doubled for the flame experiments discussed below. The spectral profile of the laser consists of several longitudinal oscillatorcavity modes $480 \mathrm{MHz}$ apart and close to Fourier transform limited in width. The few microjoule of laser light in a $2 \mathrm{~mm}$ diameter beam is coupled into 
the cavity through one of the mirrors and the time dependence of the transmitted laser intensity is monitored by a photomultiplier (PMT) placed directly behind the other cavity mirror. The PMT was placed close to the output mirror so that all of the many transverse modes were collected with equal efficiency. Beautiful beating waveforms could easily be recorded if the PMT was placed several meters from the cavity so that only the lowest divergence modes were detected. The signal from the PMT was digitized on a digital oscilloscope with a $100 \mathrm{MHz}$ sampling rate and a 10 bit vertical resolution (LeCroy 9430 ). The signal was added over a number of laser shots (typically 20 to 50 ) into a 15 bit memory at a fixed wavelength setting of the laser and the summed signal was read out by a PC. After the background was subtracted the natural logarithm of the data was taken and fitted (using a least squares weighted fitting algorithm [7]) to a straight line. The slope of this line, equal to $1 / \tau$, is conveniently expressed in ppm losses per single pass and is recorded as a function of the laser wavelength.

We first performed COCRDS measurements on a few millitorr of $I_{2}$ in order to demonstrate the high resolution afforded by the quasi-continuum mode spectrum of the cavity. The $I_{2}$ transitions have a Doppler width fwhm around $400 \mathrm{MHz}$, smaller than the $c / 4 d$ spacing of the confocal cavity (fig. 1a). The resulting spectrum is shown in fig. 2 and shows individual lines with a width of $0.05 \mathrm{~cm}^{-1}$, limited by the dye laser. Comparison with the $I_{2}$ spectral atlas [8] shows a nearly indistinguishable spectrum, in-

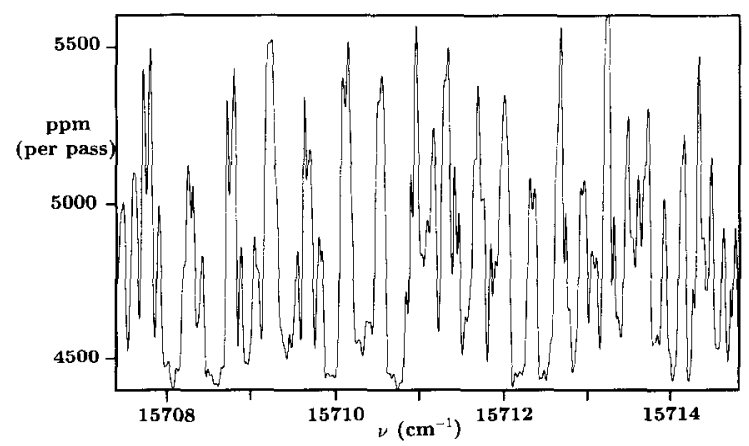

Fig. 2. Part of the room-temperature $I_{2}$ absorption spectrum as measured via coherent cavity ring down spectroscopy around 630 $\mathrm{nm}$. The width of individual lines in the spectrum is $0.05 \mathrm{~cm}^{-1}$, mainly determined by the bandwidth of the dye-laser. dicating that COCRDS delivers reliable line positions and shapes. It is important to emphasize that this spectrum could never have been recorded if the cavity mode structure were not a quasi-continuum. Typical cavity ring down times in this experiment were on the order of $100 \mathrm{~ns}$, much shorter than in previous experiments. This is a result of the short cavity design and the lower reflectivity mirrors. While this implies a loss in sensitivity, it turned out that the standard deviation in the measurement of the cavity ring down time was quite small, $0.2 \mathrm{~ns}$, translating into an absorption sensitivity of $\pm 10 \mathrm{ppm}$ per single pass.

Since it is clear that one can perform COCRDS with lower reflectivity mirrors and at higher resolution, we set out to try similar experiments in the UV. For these experiments we opened the vacuum cell and placed a $1 \mathrm{~cm}$ long slit form Bunsen burner between the mirrors. Fig. 3 shows a COCRDS absorption spectrum of $\mathrm{OH}$ in the well known $\mathrm{A}^{2} \Sigma^{+} \leftarrow \mathrm{X}^{2} \Pi$ electronic absorption system. The linewidth in this spectrum is determined by Doppler broadening; the Doppler width of $\mathrm{OH}$ at $1900 \mathrm{~K}$ is $0.25 \mathrm{~cm}^{-1}$ very close to that of fig. 3 . Assignment of the lines is made using the tables of Dieke and Crosswhite [9]. Ground state $\mathrm{OH}$ levels in the region $7000-13000 \mathrm{~cm}^{-1}$ are probed in this part of the spectrum, levels that have a relative population of

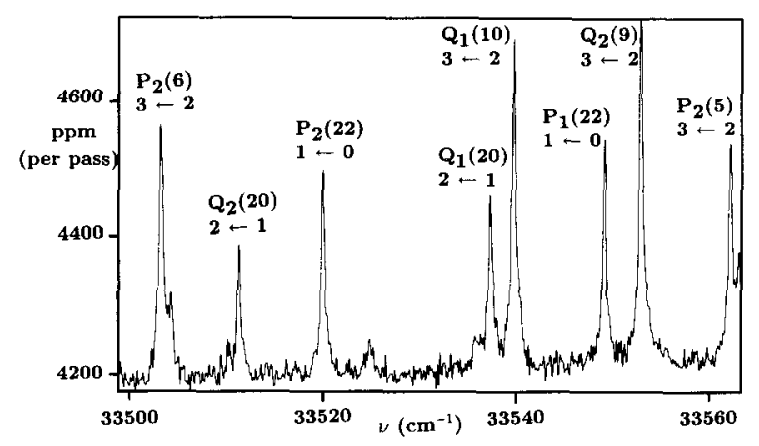

Fig. 3. Part of the $\mathrm{OH}\left(\mathrm{A}^{2} \Sigma^{+}, v^{\prime} \leftarrow \mathrm{X}^{2} \Pi, v^{\prime \prime}\right)$ absorption spectrum as measured via coherent cavity ring down spectroscopy around $298 \mathrm{~nm}$ in a $\mathrm{CH}_{4} /$ air flame. The assignment of the various lines is given in the figure. The population in $v^{\prime \prime}=0,1,2$ is probed in this region of the spectrum. The linewidth is mainly determined by Doppler broadening. The baseline is at $4200 \mathrm{ppm}$, indicating an averaged reflectivity of the two mirrors of $9958 \%$ at this wavelength $(R \geqslant 99.7 \%$ around $308 \mathrm{~nm})$. 
about $10^{-4}$ at the temperature in the flame. It should be noted that detection of the band orgin of the 0 0 vibronic band of $\mathrm{OH}$ was so easy that we even could observe a strong signal from almost thermalized $\mathrm{OH}$ ( $T_{\text {rot }} \approx 400 \mathrm{~K}$ ) some $10 \mathrm{~cm}$ above the top of the visible part of the flame, establishing a detection limit for room temperature $\mathrm{OH}$ using COCRDS of $10^{11}$ $\operatorname{radical} / \mathrm{cm}^{3}$.

As transitions were observed which probed ( $v^{\prime \prime}$, $\left.N^{\prime \prime}\right)$ levels where $v^{\prime \prime}=0,1,2$ and $4<N^{\prime \prime}<28$, it was possible to make a quantitative test of the MaxwellBoltzmann law for this flame, something that would be considerably more complicated if other detection schemes, e.g. laser-induced fluorescence, were used. The measured line intensities were normalized to correct for differences in their degeneracies $\left(2 J^{\prime \prime}+1\right)$, Hönl-London factors [9] and Franck-Condon factors. The latter are taken as $q_{2,1} / q_{1,0}=q_{3,2} / q_{1,0}=1.50$ $[10]$. The results from such a measurement are plotted in fig. 4. The fit to the data is an exponential function indicating a temperature of $1900 \mathrm{~K}$. From

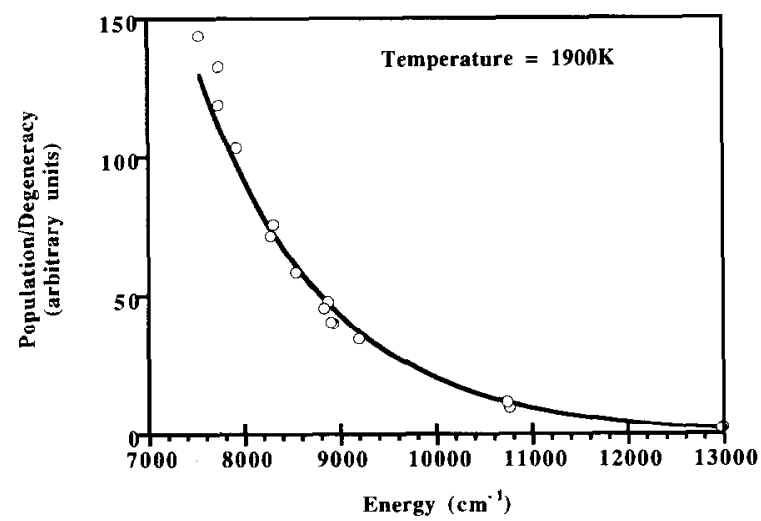

Fig. 4. The $\mathrm{OH}$ line intensities have been corrected for the degeneracy of the probed state, the Hönl-London and Franck-Condon factors of the probe transition and these values, which are proportional to the population/degeneracy, have been plotted versus the energy of the probed state (shown as open circles). The solid line is the best exponential fit to the derived population/ degeneracy values and yields a temperature of $1900 \mathrm{~K}$. the figure it is concluded that the condition of local thermodynamic equilibrium is fulfilled at least to a good approximation.

In conclusion, we have demonstrated that high sensitivity direct absorption measurements can be performed using cavity ring down in a short cavity with an almost continuous mode spectrum, thereby making a high spectral resolution possible, a technique we named COCRDS. This technique can be used from the IR into the UV and with a cavity ring down time comparable to typical radiative lifetimes of excited molecules the technique can be applied in multiple resonance experiments as well. Specifically, coherent cavity ring $u p$ as a sensitive way of detecting stimulated emission transitions should be feasible.

The visit of AMW to the Nijmegen laboratory has been made possible by financial support of the Research Institute for Materials (RIM) and the Nijmegen SON Research Center (NSR). We thank Giel Berden for his help in the measurements of the cavity mode spectrum.

\section{References}

[1] R.B. Weisman, J.I. Selco, P.O. Holt and P.A. Cahill, Rev. Sci. Instr. 54 (1983) 284.

[2] A. O'Keefe and D.A.G. Deacon, Rev. Sci. Instr. 59 (1988) 2544.

[3] A. O'Keefe, J.J. Scherer, A.L. Cooksy, R. Sheeks, J. Heath and R.J. Saykally, Chem. Phys. Letters 172 (1990) 214.

[4] D. Romanini and K.K. Lehmann, J. Chem. Phys., in press.

[5] T. Yu and M.C. Lin, J. Am. Chem. Soc. 115 (1993) 4371.

[6] H. Kogelnik and T. Li, Proc. IEEE 54 (1966) 1312.

[7] W.H. Press, B.F. Flannery, S.A. Teukolsky and W.T. Vetterling, Numerical recipes in $\mathrm{C}$; the art of scientific computing (Cambridge Univ. Press, Cambridge, 1988).

[8] S. Gerstenkorn and P. Luc, Atlas du spectroscopie d'absorption de la molecule d'iode (CNRS, Paris, 1978).

[9] G.H. Dieke and H.M. Crosswhite, J. Quantum Spectry. Radiation Transfer 2 (1962) 97.

[10] D.R. Crosley and R.K. Lengel, J. Quantum Spectry. Radiation Transfer 15 (1975) 579. 